\title{
An Evaluation of Maternal Health and Extreme Heat Exposure Training
}

\author{
Adelle Dora Monteblanco, $\mathrm{PhD}$ \\ Middle Tennessee State University, Murfreesboro, Tennessee, United States \\ University of Texas at El Paso, El Paso, Texas, United States \\ (iD https://orcid.org/0000-0001-8515-8146
}

Jennifer K. Vanos, $\mathrm{PhD}$

Arizona State University, Tempe, Arizona, United States

(iD) https://orcid.org/0000-0003-1854-9096

Sarah LeRoy, MA

University of Arizona, Tucson, Arizona, United States

Patricia M. Juárez-Carrillo, $\mathrm{PhD}$

University of Texas at El Paso, El Paso, Texas, United States

https://orcid.org/0000-0001-6180-1408

Gregg M. Garfin, $\mathrm{PhD}$

University of Arizona, Tucson, Arizona, United States

(iD https://orcid.org/0000-0002-2760-132X

Contact: adelle.monteblanco@mtsu.edu

\section{Abstract}

Growing empirical evidence documents the potential risk of extreme heat exposure to pregnant individuals. These risks include adverse birth outcomes, such as preterm birth and low birth weight. Climate change will exacerbate extreme heat exposures to a large portion of the global population, and pregnant individuals need to understand the risks and protective measures needed. Maternal health workers are a key mechanism for conveying this information to pregnant individuals. The authors assess a training of maternal health workers in El Paso, Texas, through two research instruments. First, eight maternal health workers completed an educational workshop and consented to participation; pre- and post-test workshop data are reported. Second, and subsequent to the workshop, a focus group was undertaken with six maternal health workers; directed content analysis was used to synthesize and report patterns from this qualitative data. Assessments indicated that a training session can improve provider knowledge of maternal heat-health risks and can encourage providers to discuss heat risks with patients/clients. This pilot project offered an approach to raise awareness of extreme heat among maternal health workers. The authors encourage the development of similar trainings in other

Note: The authors declare that they have no conflict of interest. This work is supported by the Agnese Nelms Haury Program in Environment and Social Justice. The authors owe much gratitude to the maternal health workers who assisted during the material design process and/or participated directly in the workshop and/or assessment. 
communities to improve the safety of pregnant individuals in warm regions and areas experiencing extreme heat.

Keywords: environmental health; extreme heat; training; health education; maternal health

Date Submitted: October 25, 2021 | Date Published: January 18, 2021

\section{Recommended Citation}

Monteblanco, A. D., Vanos, J. K., LeRoy, S., Juárez-Carillo, P. M., \& Garfin, G. M. (2021). An evaluation of maternal health and extreme heat exposure training. Journal of Social, Behavioral, and Health Sciences, 15, 31-42. https://doi.org/10.5590/JSBHS.2021.15.1.02

\section{Introduction}

In the U.S.A., more people die from the effects of heat than any other form of severe weather (National Weather Service, 2020). Global surface temperatures continue to increase due to anthropogenic climate change and urbanization (Sarofim et al., 2016; Vose et al., 2017); e.g., 2014-2018 were the warmest years in modern record (National Centers for Environmental Information, 2019), and extreme heat events in the U.S.A. are predicted to increase in frequency and intensity.

Current heat-safety outreach efforts (e.g., National Weather Service) largely target four populationschildren, outdoor workers, the elderly, and the ill. However, extreme heat exposure is also a health risk to pregnant individuals and their developing fetuses. Pregnant individuals have a compromised ability to thermoregulate (Wells, 2002), and evidence indicates that elevated apparent temperatures (or "heat index," a combination of temperature and humidity) can exacerbate the risk of many adverse fetal outcomes, including stillbirth, preterm delivery, and lower birth weight (Barreca \& Schaller, 2020; Basu et al., 2016; Beltran et al., 2013; Molina \& Saldarriaga, 2017; Ward et al., 2019). As pregnant individuals are increasingly exposed to high temperatures, these heat-related adverse outcomes may become more common, exacerbating our current infant and maternal health crisis (Kassebaum et al., 2016; MacDorman et al., 2014).

Particular geographies, including the U.S. Southwest (Gonzalez et al., 2018; Guirguis et al., 2018) and U.S.-Mexico border cities, such as El Paso, Texas, are particularly vulnerable to heat extremes (Wilder et al., 2013). In 2015, El Paso County recorded 106 consecutive days above $90^{\circ} \mathrm{F}$ (Garfin et al., 2017) and reported 72 heat-related deaths from 2002 to 2016 (Borunda, 2016). El Paso's social and economic circumstances compound this vulnerability. ${ }^{1} \mathrm{El}$ Paso and its sister border city, Ciudad Juárez, Chihuahua, Mexico, have high poverty rates (22\% of area residents live below poverty), low access to medical care (34\% of adults lack health insurance; Healthy Paso del Norte, 2017), high rates of substandard housing (less likely to have working air conditioning, may work high-heat jobs), and a large transient population (less likely to be heat acclimatized; Grineski et al., 2012).

The increased risk of adverse birth outcomes and the geographic, social, and economic vulnerabilities indicate a need for a targeted training program to confirm knowledge or teach healthcare workers about these health issues and to encourage their pregnant clients to take precautions. Although many scholars have urged for the healthcare community to address the challenges of climate change (Parker et al., 2020), no studies have specifically sought to create and test the effects of heat-health education for maternal and fetal health providers.

\footnotetext{
${ }^{1}$ Although beyond the scope of this study, El Paso county is over 1,000 square miles; like other cities across the globe, residents of different neighborhoods likely perceive and experience environmental threats in different ways (Mason et al., 2017).
} 


\section{History of Training Development}

As one part of a larger effort to increase community resilience to the public health risks of extreme heat events along the U.S.-Mexico border, the authors developed a pilot program (with outreach materials, a training workshop, and a concluding focus group) for community-based maternal health workers (MHWs) in the Paso del Norte region. We aimed for a broad classification of MHWs because every health provider-even those who work outside of a hospital setting, such as direct-entry midwives (Monteblanco \& Leyser-Whalen, 2019)-should be able to prepare their clients for environmental hazards. MHWs were the focus of this pilot project because they are trusted providers who often have multiple visits with their clients during pregnancy and post-partum and are already managing numerous roles: medical expert, communicator, and health advocate (Fontein-Kuipers et al., 2018). Thus, MHWs are ideally positioned to support pregnant individuals' heat safety by disseminating health information and materials.

\section{Training Development}

As reported elsewhere (Monteblanco \& Vanos, 2020), the first two authors collected and analyzed 12 semi-structured interviews with MHWs serving the region. Participants were asked about how they perceived heat risk, what they knew about extreme heat vulnerability and protective measures, and which prenatal consultation topics they prioritized. Results indicate that participants had little concern about heat exposure and limited knowledge of heat-health vulnerabilities and risks to pregnant individuals. However, participants were quite familiar with heat-protective measures and eager for more information.

\section{Training Implementation, Setting, and Recruitment}

In response to the above identified interview themes, the authors developed a two-hour training titled "Heat Exposure and Maternal Health." The flyer described it as an "extreme heat training workshop which aims to promote maternal and infant health in the region." The goals were to raise MHWs' awareness about the adverse birth outcomes associated with high heat exposure, encourage dialogue of shared experiences, and to discuss preventative measures. The training sought to educate participants in two categories: understanding climate and heat risk and understanding how MHWs can help protect their clients. Climate-related learning objectives were to: (1) define normal and extremely hot temperatures for El Paso, (2) learn where to find information about weather and weather forecasts, and (3) understand terms used by the National Weather Service in preparation for abnormally hot days. Maternal healthrelated learning objectives were to (1) understand the different ways high temperatures affect pregnant individuals, fetuses, nursing women, and infants, and (2) list El Paso resources to keep pregnant individuals and babies cool during high temperatures and ways to reduce exposure.

The workshop targeted a wide range of MHWs-those providing direct clinical care and those offering other related services to infants and/or pregnant individuals-in the Paso del Norte region, including El Paso, TX, Las Cruces, New Mexico, and Ciudad Juárez, Mexico. Recruitment occurred through word of mouth, flyers posted to the social media accounts of local doulas and birth centers, and flyers disseminated at local maternal and infant health events. Workshop attendees were provided a $\$ 10$ incentive and breakfast snacks. This training was held in April 2018 in the community room of an El Paso freestanding birth center. While the birth center hosts numerous community and educational events, the location likely shaped attendance and may have deterred hospital-based providers because of the stigma associated with out-of-hospital birth (Monteblanco, 2018).

\section{Outreach Materials}

One goal of the training was to offer evidence-based, effective outreach materials in both English and Spanish for MHWs to take with them and share with their clients. To create these materials, the authors 
consulted the literature and two local MHWs during the design process. The materials, produced by a graphic designer, included a rack card, a urine chart, a thermometer, a magnet, and a pamphlet. The rack card (Figure 1) offered a quick reminder to stay safe during high temperatures. The urine chart (Figure 2), similar to visuals used by the military and the healthcare industry, encouraged hydration. ${ }^{2}$ The small thermometer (not shown) was designed to be added to a keychain or carried in a car. The magnet (not shown) offered quick tips to "beat the heat," such as "seek shade and cooling" and "protect your loved ones." The pamphlet (not shown) detailed ways to reduce home temperatures using passive cooling-such as shade sails or improving insulation-to promote low-energy and low-cost methods of cooling family homes.

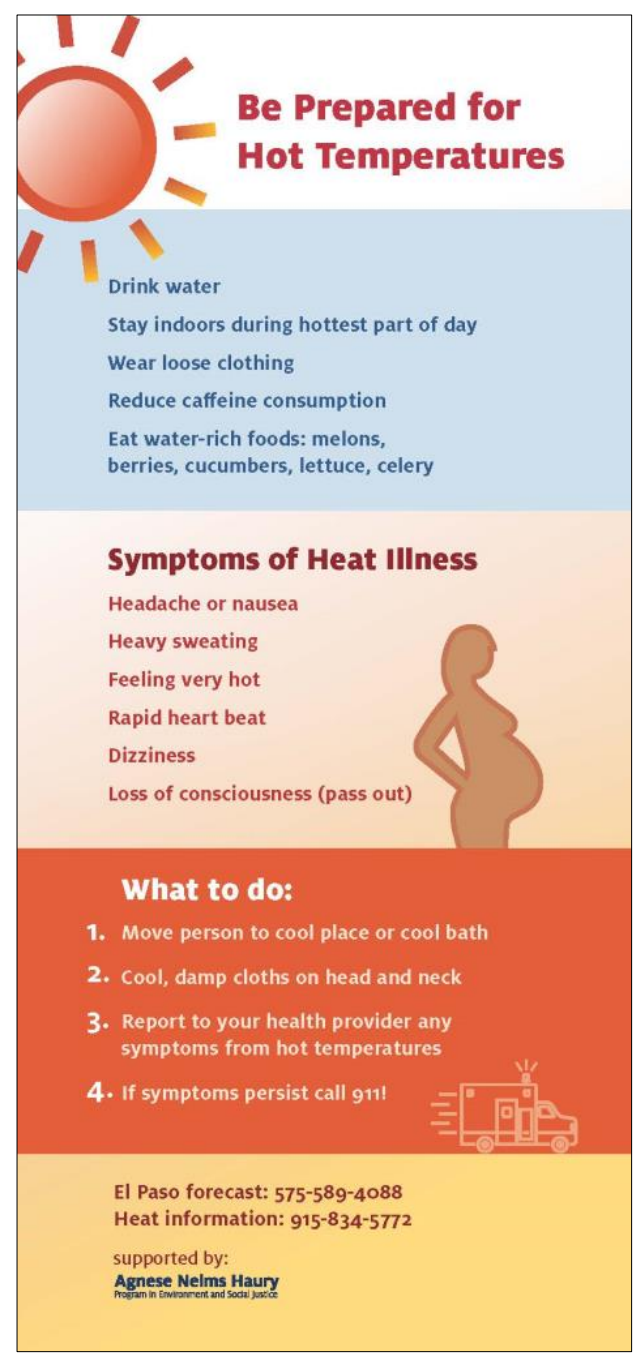

Figure 1: Rack Card

${ }^{2}$ A few months later, two participants asked for more rack cards and urine charts. 


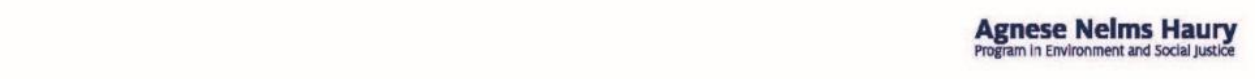

Hydration Urine Chart

Check the color of your urine as a good indicator of your hydration status.
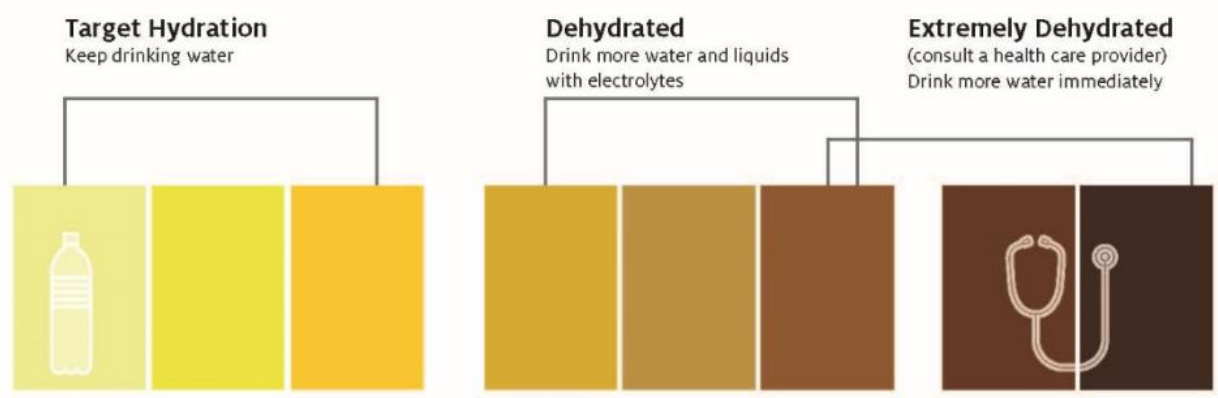

*This color chart is not for clinical use.

It is important to note that some food, vitamins, and medications can change the color of your urine.

Figure 2: Urine Chart

\section{Methods}

\section{Data Collection}

A short demographic survey collected participant information. Eight of the 10 workshop participants consented to the collection of pre-/post-survey data assessing knowledge gain. Seven survey questions were asked. Most were statements that asked respondents to mark as "True," "False," or "I don't know." For example, participants were given those three response options after the following statements: "Certain medications can make someone more vulnerable to heat stress" and "Exposure to unusually hot temperature increases the risk of preterm birth."

The majority of our reported data relied on qualitative collection methods. The researchers held a onehour focus group, seven months after the workshop, in the same El Paso location. Led by author Leroy, participants were asked to reflect on the 2018 heat season, the workshop, their use of the educational materials, and additional resources needed to promote healthy pregnancies in extreme heat areas. Participants were offered lunch, but no cash incentive.

\section{Data Analysis}

Paired sample $t$-tests were not conducted for question sub-scales because of the small number of consenting workshop participants $(N=8)$. However, we offer brief descriptive statistics in the "Results" section below. Directed content analysis was used to analyze focus group data (Hsieh \& Shannon, 2005). In repeatedly reading the transcript line-by-line, we identified key patterns and organized them into coding and sub-coding categories. This effort helped identify themes and quotes by which to clarify the themes. Data collection and analysis were approved by the University of Texas at El Paso Institutional Review Board. 


\section{Results}

\section{Participant Characteristics}

The workshop participants who consented to data collection have the following demographic characteristics: all identified as women; four identified as Hispanic and four as non-Hispanic (three as white, one as Jewish American); three were aged 30 or younger, two were 31-40, and three were 50-65 years old. Participants had completed various levels of education: one reported a high school diploma, one reported an associate's degree, four reported a bsachelor's degree, and two reported graduate degrees. The demographic survey asked participants what they identify as their "dominant profession," because they often have many work roles; three participants identified as midwives, one identified as a doula, one identified as both a doula and lactation counselor, two identified as lactation consultants, and one identified as a student studying midwifery. In these roles, they serve in homes, birth centers, and/or hospitals.

We did not collect separate demographic information at the focus group, but the six participants (four of them part of the original workshop group of eight) present similar identities across race, age, education, and work roles. 3

\section{Reported Motivation for Attendance at Training}

At the beginning of the training, the participants were asked, "What motivated you to participate in this workshop?" They could circle as few or as many options as desired from a list that included "topical interest" (selected by 7), "a colleague invited you" (selected by 6), "opportunity to earn CEUs [Continuing Education Units]"4 (selected by 4), "opportunity to network" (selected by 3), and/or "the \$10 incentive" (selected by none). The option of "other" was selected by one individual, who wrote in: "support research in El Paso."

\section{Training: Assessed Knowledge Gain}

The pre-survey included seven questions; the eight participants answered all of them for a total of 56 answers. Of these 56 questions, 17 ( $30 \%)$ were answered incorrectly or with the answer, "I don't know." As one example, in the pre-survey, participants were given the statement, "Exposure to unusually hot temperature increases the risk of low birth weight” with the potential to check “True," "False," or "I don't know"; four of eight participants answered, "I don't know" although the correct answer was "True." As a second example, in the pre-survey, participants were given the statement, "Exposure to unusually hot temperature increases the risk of preterm birth" with the potential to check “True," "False," or "I don't know"; three of eight participants answered "I don't know" although the correct answer was "True." However, the majority of responses were correct in the other pre-survey questions. For example, using the same question format and three responses, the statements "Exposure to unusually hot temperature increases the risk of fetal death" and "Certain medications can make someone more vulnerable to heat stress" were correctly marked "True" by the majority of participants. After participants completed the

\footnotetext{
${ }^{3}$ Four of the focus group attendees were recruited from the original workshop, and two others attended based on informal conversations with the first author. Importantly, these two participants did not complete the prior workshop but they reviewed the electronic files associated with the workshop, including the PowerPoint slides, and were provided with all outreach materials; they consented to research participation at the focus group.

${ }^{4}$ The authors did not organize the workshop to provide CEUs, because the variety of MHWs participating meant a diversity of associated licenses, certificates, and state/country differences (e.g., New Mexico vs. Texas). A nurse-midwife participant helped workshop attendees earn CEUs.
} 
workshop, only one question was answered incorrectly (less than $2 \%$ ), and no question was answered with "I don't know."

\section{Focus Group: Reported Satisfaction with Training}

During the focus group, the six participants expressed satisfaction with the workshop and the educational outreach materials. Five participants said that this training was the first time they had learned of the health risks that extreme heat exposure poses to pregnant individuals. For example, a doula (who did not attend the workshop) said, "I know heat was bad for people and their health but I wasn't fully aware of the true effects it could cause on the body, especially for more vulnerable populations." 5

The focus-group participants recounted frequent use of the rack cards and urine charts. A midwife student (who did attend the workshop) reported that the urine chart was posted in the bathroom of one birth center; a lactation consultant (who did attend the workshop) commented that the thermometers were "really popular [at her breastfeeding support group] and it did get the conversation started [with parents]."

Many focus group participants reported important changes in post-workshop behavior. Promoting hydration, a lactation consultant (who did attend the workshop), who oversees the breastfeeding support group mentioned previously, stated, "We went out and bought that Brita filtered water container and drag it into the space every week now, after the presentation." Many participants reported using the outreach materials, especially the rack card, to spur consultations with their clients. One doula (who did not attend the workshop) said, "It would open the conversation of like being sun smart. Like walk early in the day, walk later in evening, don't overexert yourself. If you're going to go outside, take water with you, things like that."

The participants noted that they adapt the materials to the season and to the needs and knowledge of each patient or client. Seasonality matters in the arid southwest, and participants reported using the outreach materials only between April and October in 2018. El Paso has a large military community, and the participants reported prioritizing heat-health conversations with military spouses new to the region, who are often ill prepared for the summer heat. Evidence shows MHWs prioritize heat information based on time and clientele, while also navigating a diversity of consultation topics and fielding numerous questions during the pregnancy and/or post-partum phases. The authors anticipate that the information will easily fit into conversations on general nutrition or warning signs of illness.

The participants noted limitations: the magnet and passive cooling brochure were reported to be the least helpful of the educational materials. The passive cooling brochure was less useful for several reasons: because the focus group participants worked at birth centers or nonprofits rather than in people's homes, because many of their clients had and could afford AC, and because they were not comfortable suggesting changes to families' homes. However, such brochures could be helpful for MHWs that do visit clients' homes.

\section{Ongoing Efforts}

During the focus group, the participants offered recommendations. They requested that the pregnancyfocused rack card include additional information, such as recommended water intake and safety reminders for walking outside. Focus group participants also requested the creation of a separate heatrelated handout targeting post-partum parents with reminders about breastfeeding, hydration, and infant

\footnotetext{
${ }^{5}$ Quotes are shared without edits.
} 
heat safety. ${ }^{6}$ The focus group also discussed the benefit of a "cheat-sheet" for providers, which would enable them to answer clients' questions about heat exposure, climate change, etc. Participants had been provided with a map of the El Paso cooling centers; because residents of the Paso del Norte region are highly mobile, participants also requested a map of Cd. Juarez's cooling centers.

\section{Discussion and Conclusion}

The climate change and public health literatures indicate that extreme heat events are becoming more frequent and severe across the globe (Vose et al., 2017). It is well known that children, outdoor workers, the elderly, and the ill are physiologically vulnerable to heat exposure. However, another vulnerable population-pregnant individuals-is not often targeted by public health campaigns or heat warning messaging (Ward et al., 2019).

Pregnant individuals should be educated about this vulnerability and given recommendations for reducing their heat exposure. Maternal health workers are a key mechanism for conveying this information (Basu et al., 2015; Kuehn \& McCormick, 2017). However, MHWs need continuing education and outreach to contribute to their community's heat resilience in this way. A review of the existing literature indicates no formal heat-focused training targeting this population of MHWs. Thus, our workshop was unique in its participants, location, and goals.

This research effort first interviewed local MHWs (Monteblanco \& Vanos, 2020). Our interviews indicated MHWs' limited knowledge of heat as a health risk to pregnant individuals. We used this information to design and assess the workshop in the current study. The pre- and post-surveys showed that the workshop-participating MHWs started with a relatively high rate of knowledge and gained additional knowledge during the workshop. Importantly, selection bias likely shaped the survey success based on who wanted to and could attend the workshop and because the questions were designed to be relatively simple so as not to intimidate participants.

Our findings from a focus group seven months subsequent to the training further illustrated the value of this brief education session to develop and mobilize knowledge. Focus group participants reported that the workshop improved their knowledge and attention to heat; that they had begun to incorporate heat conversations with clients; and that they found many of the outreach materials useful.

The brochure for passive cooling was reported to be less useful. We were disappointed that participants were disinterested in or uncomfortable with the idea of promoting passive cooling to their clients, yet such advice is home-specific and may work in at-home consultation settings lacking air conditioning. We will explore other ways to encourage pregnant individuals and their families to implement these strategies; we plan to particularly target lower-income families who are less likely to have air conditioning (and thus benefit from passive cooling).

Providing training to MHWs about environmental health, and specifically heat-related threats, can improve the safety of pregnant individuals in warm regions and areas experiencing extreme heat throughout the year. Results from a pilot study indicate that a short training session can improve provider knowledge of maternal heat-health risks and can encourage providers to discuss heat risks with patients/clients (henceforth clients). This descriptive study aims to encourage the development of similar training in other communities.

\footnotetext{
${ }^{6}$ Breastfeeding USA recently created an infographic titled, "Summertime Breastfeeding" that is available at https://www.facebook.com/BreastfeedingUSA
} 
Lastly, this study inspires numerous policy changes. The authors encourage the creation of more climate change, environmental health, and extreme events-related curriculum embedded in MHWs' formal training; the targeted MHWs should include those participating in this project, such as midwives, doulas, lactation consultants, and lactation counselors, as well as childbirth educators and physicians. The curriculum topics should broadly cover environmental health and focus on risks relevant to local geography and clientele. As evident in this pilot project, access to training on the risks that extreme heat poses to fetuses and pregnant individuals can develop the skills to offer recommendations and resources that may mitigate heat risk. An informed maternal healthcare sector can promote community health through extreme heat preparedness. 


\section{References}

Barreca, A., \& Schaller, J. (2020). The impact of high ambient temperatures on delivery timing and gestational lengths. Nature Climate Change, 1O(1), 77-82. https://doi.org/10.1038/s41558-0190632-4

Basu, R., Pearson, D., Sie, L., \& Broadwin, R. (2015). A case-crossover study of temperature and infant mortality in California. Paediatric and Perinatal Epidemiology, 29(5), 407-415. https://doi.org/10.1111/ppe.12204

Basu, R., Sarovar, V., \& Malig, B. J. (2016). Association between high ambient temperature and risk of stillbirth in California. American Journal of Epidemiology, 183(10), 894-901. https://doi.org/10.1093/aje/kwv295

Beltran, A. J., Wu, J., \& Laurent, O. (2013). Associations of meteorology with adverse pregnancy outcomes: A systematic review of preeclampsia, preterm birth and birth weight. International Journal of Environmental Research and Public Health, 11(1), 91-172. https://doi.org/10.3390/ijerph110100091

Borunda, D. (2016, July 9). Health officials: Heat wave kills 4 in El Paso. El Paso Times. http://www.elpasotimes.com/story/weather/2016/07/19/health-officials-heat-wave-kills-4-elpaso/87319092

Fontein-Kuipers, Y., Romeijn, E., Zwijnenberg, A., Eekhof, W., \& van Staa, A. (2018). 'ISeeYou': A woman-centred care education and research project in Dutch bachelor midwifery education. Health Education Journal, 77(8), 899-914. https://doi.org/10.1177/0017896918784618

Garfin, G., LeRoy, S., \& Jones, H. (2017). Developing an integrated heat health information system for long-term resilience to climate and weather extremes in the El Paso-Juárez-Las Cruces region: Report from the workshop held in El Paso, TX, July 13, 2016. Tucson, AZ. https://doi.org/http://doi.org/10.7289/V5930R6Q

Gonzalez, P., Garfin, G. M., Breshears, D. D., Brooks, K. M., Brown, H. E., Elias, E. H., Gunasekara, A., Huntly, N., Maldonado, J. K., Mantua, N. J., Margolis, H. G., McAfee, S., Middleton, B. R., \& Udall, B. H. (2018). In D. R. Reidmiller, C. W. Aver, D. R. Easterling, K. E. Kunkel, K. L. M. Lewis, T. K. Maycock, \& B. C. Stewart (Eds.), Impacts, risks, and adaptation in the United States: Fourth national climate assessment, Volume II (pp. 1101-1184). U.S. Global Change Research Program. https://doi.org/10.7930/NCA4.2018.CH25

Grineski, S. E., Collins, T. W., Ford, P., Fitzgerald, R., Aldouri, R., Velázquez-Angulo, G., Romo Aguilar, \& $\mathrm{Lu}, \mathrm{D}$. (2012). Climate change and environmental injustice in a bi-national context. Applied Geography, 33, 25-35. https://doi.org/10.1016/j.apgeog.2011.05.013

Guirguis, K., Gershunov, A., Cayan, D. R., \& Pierce, D. W. (2018). Heat wave probability in the changing climate of the Southwest US. Climate Dynamics, 5o(9-10), 3853-3864. https://doi.org/10.1007/s00382-017-3850-3

Healthy Paso del Norte. (2017). July 1, 2018. www.healthypasodelnorte.org

Hsieh, H.-F., \& Shannon, S. E. (2005). Three approaches to qualitative content analysis. Qualitative Health Research, 15(9), 1277-1288. 
Kassebaum, N. J., Barber, R. M., Bhutta, Z. A., Dandona, L., Gething, P. W., Hay, S. I., Kinfu, Y., Larson, H. J., Liang, X., Lim, S. S., Lopez, A. D., Lozano, R., Mensah, G. A., Mokdad, A. H., Naghavie, M., Pinho, C., Salomom, J. A., Steiner, C., Vos, T., ... Murray, C. J. L. (2016). Global, regional, and national levels of maternal mortality, 1990-2015: a systematic analysis for the Global Burden of Disease Study 2015. The Lancet, 388(10053), 1775-1812. https://doi.org/10.1016/So1406736(16)31470-2

Kuehn, L., \& McCormick, S. (2017). Heat exposure and maternal health in the face of climate change. International Journal of Environmental Research and Public Health, 14, 853. https://doi.org/10.3390/ijerph14080853

MacDorman, M. F., Mathews, T. J., Mohangoo, A. D., \& Zeitlin, J. (2014). National vital statistics reports international comparisons of infant mortality and related factors: United States and Europe, 2010. National Vital Statistics Reports, 63(5), 1-6. https://www.cdc.gov/nchs/data/nvsr/nvsr63/nvsr63_05.pdf

Molina, O., \& Saldarriaga, V. (2017). The perils of climate change: In utero exposure to temperature variability and birth outcomes in the Andean region. Economics and Human Biology, 24, 111124. https://doi.org/10.1016/j.ehb.2016.11.009

Monteblanco, A. D. (2018). Midwives on the margins: Stigma management among out-of-hospital midwives. Deviant Behavior, 39(12), 1615-1632. https://doi.org/10.1080/01639625.2017.1410624

Monteblanco, A. D., \& Leyser-Whalen, O. (2019). Thinking outside of the hospital and nurse-midwife paradigms: A qualitative examination of midwifery in times of natural disasters. International Journal of Mass Emergencies and Disasters, 37(2), 138-173.

Monteblanco, A., \& Vanos, J. K. (2020). Community-based maternal health workers' perspectives on heat risk and safety: A pilot qualitative study. Health Care for Women International, 1-21. https://doi.org/10.1080/07399332.2020.1803873

National Centers for Environmental Information. (2019). Assessing the global climate in 2018. https://www.ncei.noaa.gov/news/global-climate-201812

National Weather Service (2020). Weather Fatalities 2018.https://www.weather.gov/hazstat/

Parker, G., Berta, W., Shea, C., \& Miller, F. (2020). Environmental competencies for healthcare educators and trainees: A scoping review. Health Education Journal, 79(3), 327-345.

Mason, R. L., Ellis, K. N., \& Hathaway, J. M. (2017). Experiences of urban environmental conditions in socially and economically diverse neighborhoods. Journal of Community Practice, 25(1), 48-67.

Sarofim, M. C., Saha, S., Hawkins, M. D., Mills, D. M., Hess, J., Kinney, P., Schwartz, J., \& St Juliana, A. (2016). Temperature-related death and illness. In A. Crimmins, J. Balbus, J. L. Gamble, C. B. Beard, J. E. Bell, D. Dodgen, R. J. Eisen, N. Fann, M. D. Hawkins, S. C., Herring, L. Jantarasami, D.M. Mills, S. Saha, M.C. Sarofim, J. Trtanj, and L. Ziska, Eds. The impacts of climate change on human health in the United States: A scientific assessment. U.S. Global Change Research Program, pp. 43-68. https://doi.org/10.7930/JoMG7MDX

Vose, R. S., Easterling, D. R., Kunkel, K. E., LeGrande, A. N., \& Wehner, M. F. (2017). Climate science special report: Fourth national climate assessment (Vol. 1). In D. J. Wuebbles, D. W. Fahey, A. Hibbard, D. J. Dokken, B. C. Stewart, \& T. K. Maycock (Eds.) (pp. 185-206). Washington, DC, USA. 
Ward, A., Clark, J., McLeod, J., Woodul, R., Moser, H., \& Konrad, C. (2019). The impact of heat exposure on reduced gestational age in pregnant women in North Carolina, 2011-2015. International Journal of Biometeorology, 63(12), 1611-1620.

Wells, J. C. K. (2002). Thermal environment and human birth weight. Journal of Theoretical Biology, 214(3), 413-425. https://doi.org/10.1006/jtbi.2001.2465

Wilder, M., Garfin, G., Romero-Lankao, P., Lara-Valencia, F., Cortez-Lara, A. A., Muñoz-Arriola, F., \& Varady, R. G. (2013). Climate change and U.S.-Mexico border communities. In G. Garfin, A. Jardine, R. Merideth, M. Black, \& S. LeRoy (Eds.), Assessment of climate change in the southwest United States: A report prepared for the National Climate Assessment. Southwest Climate Alliance. 